\title{
Transport of Palladium Nanoparticles in Porous Media of Varying Grain Size with Synchrotron X-Ray Computerized Tomography
}

E.M. SAURETTE ${ }^{1}$ A. LenTZ ${ }^{1}$ S. LINLEY ${ }^{2}$, X.F G G $^{2}, \mathrm{M}$. RIVERS $^{3}$, D.W. BLOWES ${ }^{1}$, C.J. PTACEK ${ }^{1}$

${ }^{1}$ Department of Earth and Environmental Sciences, University of Waterloo, Waterloo, Ontario, Canada, N2L 3G1, emily.saurette@uwaterloo.ca, alentz@uwaterloo.ca, blowes@uwaterloo.ca,ptacek@uwaterloo.ca

${ }^{2}$ Department of Chemical Engineering, University of Waterloo, Waterloo, Ontario, Canada, N2L 3G1, stuart.linley@uwaterloo.ca, frank.gu@uwaterloo.ca

${ }^{3}$ Center for Advanced Radiation Sources, University of Chicago, 9700 South Cass Avenue, Building 434A, Lemont, Illinois 60439, United States, rivers@cars.uchicago.edu

Understanding the transport of nanoparticles (NPs) in porous media is a fundamental aspect of assessing the fate of NPs in the environment, both as emerging contaminants and as remediation agents. The properties of the porous media, aqueous solution, and NPs all affect the transport of NPs in subsurface environments. In this study, transport of Pd-NP suspended in aqueous solution (ultrapure water; UPW, or simulated groundwater; SGW) through porous media (Ottawa sand, Ottawa sand + clay, or Borden sand) was evaluated in column experiments using traditional effluent breakthrough curves (BTC) and nondestructive synchrotron x-ray computed microtomography (SXCMT) imaging analyses. Inverse modelling using the computer code CXTFIT STANMOD was used to inversely determine dispersion coefficients (D) and retardation factors $(\mathrm{R})$ from the BTC data for each column. Nanoparticle transport was more retarded in porous media with finer particle size, or, higher particle surface area, and greater mineralogical diversity (Borden sand) compared to uniform silica sand (Ottawa sand). The retardation of NPs also increased with simulated groundwater as the carrier solution. Five three-dimensional images were collected for each column using SXCMT difference imaging which provided estimates of aqueous Pd NP concentrations in thin slice sections and in individual pore spaces. SXCMT difference imaging was used to evaluate the concentration of NP within porous media at the pore scale to facilitate understanding of NP transport processes and show that the Pd concentration distributions vary with time and the properties of the pore space. 